Research Paper

\title{
Circular RNA hsa_circ_0000700 promotes cell proliferation and migration in Esophageal Squamous Cell Carcinoma by sponging miR-1229
}

\author{
Jun Fang1\#, Wen Hao Ji1\#, Fang Zheng Wang1,2, Tie Ming Xie ${ }^{3}$, Lei Wang1,2, Zhen Fu Fu1,2, Zhun Wang1, \\ Feng Qin Yan ${ }^{1,2}$, Qi Liang Shen ${ }^{1}$, Zhi Min Ye ${ }^{1,2 \bowtie}$ \\ 1. Department of Radiotherapy, The Cancer Hospital of the University of Chinese Academy of Sciences (Zhejiang Cancer Hospital), Institute of Basic Medicine \\ and Cancer (IBMC), Chinese Academy of Sciences, Hangzhou, Zhejiang, 310022, China. \\ 2. Key Laboratory of Head \& Neck Cancer Translational Research of Zhejiang Province, Hangzhou, Zhejiang, 310022, China. \\ 3. Department of Radiology, The Cancer Hospital of the University of Chinese Academy of Sciences (Zhejiang Cancer Hospital), Institute of Basic Medicine \\ and Cancer (IBMC), Chinese Academy of Sciences, Hangzhou, Zhejiang, 310022, China. \\ \#Co-first authors.
}

$\triangle$ Corresponding author: Zhi Min Ye, Institute of Cancer and Basic Medicine (ICBM), Chinese Academy of Sciences, Hangzhou, China; Department of Radiology, Cancer Hospital of the University of Chinese Academy of Sciences, Hangzhou, China; Department of Radiology, Zhejiang Cancer Hospital, Hangzhou, China; Key Laboratory of Head \& Neck Cancer Translational Research of Zhejiang Province, Hangzhou, China. No. 1, Banshan East Road, Gongshu District, Hangzhou, 310022, China. E-mail: yezm@zjcc.org.cn.

(c) The author(s). This is an open access article distributed under the terms of the Creative Commons Attribution License (https://creativecommons.org/licenses/by/4.0/). See http://ivyspring.com/terms for full terms and conditions.

Received: 2020.04.17; Accepted: 2021.02.15; Published: 2021.03.05

\begin{abstract}
Accumulating evidence has demonstrated that circular RNAs (circRNAs) are involved in the pathogenesis of cancer, including that of esophageal squamous cell carcinoma (ESCC). The current study aimed to investigate the role of hsa_circ_0000700 in ESCC. hsa_circ_0000700, miR-1229, and related functional gene expression was measured by RT-qPCR. To characterize the functions of hsa_circ_0000700 and miR-1229, ESCC cells were infected with hsa_circ_0000700-specific siRNA, miR-1229 mimics, and an inhibitor alone or in combination. Cell Counting Kit-8 (CCK8), colony formation, EdU, flow cytometry, and Transwell assays were employed to evaluate cell proliferation, apoptosis, and migration. Luciferase reporter and RNA immunoprecipitation assays were used to confirm the targeting relationship between hsa_circ_0000700 and miR-1229. Finally, a competing endogenous RNAs (ceRNA) network was built for hsa_circ_0000700, and miR-1229 targets were analyzed by bioinformatics. circ_0000700 expression was significantly upregulated in ESCC cell lines. Actinomycin D and RNase R treatment confirmed that circ_0000700 was more stable than its linear $\mathrm{CDH} 9$ mRNA form. Moreover, a cytoplasmic and nuclear fractionation assay suggested that circ_0000700 was mainly distributed in the cytoplasm of ECA-109 and TE-1 cells. In vitro, the proliferative and migratory capacities of ECA-109 and TE-1 cells were inhibited by knocking down circ_0000700 expression. Additionally, miR-1229 silencing reversed the circ_0000700-specific siRNA-induced attenuation of malignant phenotypes. Mechanistically, circ_0000700 was identified as a sponge of miR-1229 and could activate PRRG4, REEP5, and PSMB5 indirectly to promote ESCC progression. In summary, our results suggest that hsa_circ_0000700 functions as an oncogenic factor by sponging miR-1229 in ESCC.
\end{abstract}

Key words: esophageal squamous cell cancer; hsa_circ_0000700; hsa_miR-1229; cell proliferation; cell migration

\section{Introduction}

Esophageal carcinoma (EC) is the $7^{\text {th }}$ most common malignant digestive tract cancer, ranking $6^{\text {th }}$ in mortality overall, with an estimated 572,000 new cases diagnosed and more than 509,000 deaths in 2018 worldwide, with the latter meaning that EC accounts for an estimated 1 in every 20 cancer-related deaths [1, 2]. Histologically, most esophageal cancers are divided into esophageal adenocarcinoma (EAC) and esophageal squamous cell cancer (ESCC). ESCC is the most common histological type of EC [3]. Among the EC cases, 90\% are ESCC, which seriously threatens human health in developing countries [4]. Currently, surgical treatment, chemotherapy, radiotherapy, and targeted molecular therapy for cancers are developing 
increasingly rapidly and can contribute to improving the outcomes of ESCC patients, but the 5-year survival rate of patients with ESCC is still less than $13 \%[4,5]$. There are no obvious clinical features in the early stages of ESCC, resulting in the majority of ESCC patients having lost the opportunity for surgical treatment at the time of initial diagnosis. However, the exact diagnosis, prognostic prediction and underlying mechanism of esophageal cancer progression remain to be further elucidated. Therefore, there is an imperative need to identify novel sensitive biomarkers and therapeutic molecules to facilitate effective ESCC diagnosis and treatment.

Circular RNAs (circRNAs) are a particular type of endogenous noncoding RNA (ncRNA) characterized by a covalent closed-loop structure without a 5' cap or 3' tail and are more stable and free from degradation by RNA exonucleases than traditional linear RNAs [6, 7]. In addition, it is noteworthy that the biological function of circRNAs is highly associated with specific tissues, cell types, and disease stages, making circRNAs promising noninvasive biomarkers for the diagnosis and prognosis of cancer patients. Many studies have demonstrated that circRNAs play a crucial role in the regulation of cellular biological events, including proliferation, apoptosis, and metastasis [5, 8]. However, the biological functions and potential mechanisms of dysregulated circRNAs in the initiation and development of ESCC remain mostly unclear.

MicroRNAs (miRNAs) are endogenous, small ncRNA molecules that are 18-24 nucleotides in length and negatively regulate gene expression via base pairing with the 3 '-untranslated region (3'-UTR) of target genes [9]. Increasing evidence has demonstrated that circRNAs play an essential role as miRNA sponges via a competitive endogenous RNA (ceRNA) mechanism in many human cancers to attenuate the inhibitory effects of miRNAs on their target genes. For instance, $\mathrm{Xu}$ et al. showed that circ_0000654 facilitates ESCC cell proliferation, migration, invasion, and apoptosis in vitro by sponging miR-149-5p [10], and Huang et al. reported that the upregulation of circular RNA ciRS-7 expression enhances the migration and invasion of ESCC cells by affecting miR-7/KLF4 and NF-KB signaling [11]. Likewise, the circRNA hsa_circRNA_ 100367 promotes KYSE-150R cell proliferation, migration, and radioresistance by binding to miR-217 and can serve as a promising biomarker and potential therapeutic target [12]. These circRNA-miRNA signaling axes are related to the comprehensive malignant characteristics of ESCC, such as cancer cell apoptosis, metastasis, invasion, and radioresistance, which implies the potential value of circRNAs as novel therapeutic targets and biomarkers in ESCC.

With the development of high-throughput sequencing, although circRNAs were first discovered in 1976, a growing number of circRNAs have been confirmed to be linked to the progression of diverse malignancies, including ESCC. Similarly, Shi et al. observed 469 upregulated circRNAs and 275 downregulated circRNAs in ESCC by using the high-throughput Arraystar Human circRNA Array [13]. In addition, Song et al. revealed that hsa_circ_0000337 (known as hsa_circRNA_100872), one of the top ten upregulated circRNAs, promotes cell proliferation, migration, and invasion in ESCC [7].

The novel circRNA hsa_circ_0000700 (gene symbol CHD9, also known as hsa_circRNA_001937), which was found to be remarkably upregulated with a fold change $(\mathrm{FC})=6.517$ and $P$-value $=0.015$ and is located at chr16:53155459-53155541, attracted our attention. Our study aimed to investigate the expression of hsa_circ_0000700; evaluate its roles in cell proliferation, apoptosis, and metastasis in vitro; and confirm that circ_0000700 functions as a ceRNA by sponging miR-1229 in ESCC cells. Our present study found that circ_0000700 expression was significantly upregulated in the ECA-109 and TE-1 cell lines. Additionally, we also demonstrated that siRNA knockdown of circ_0000700 expression and overexpression of miR-1229 inhibited the proliferation and migration of ECA-109 and TE-1 cells. Mechanistically, circ_0000700 was found to exacerbate ESCC progression via regulation of miR-1229/ PRRG4/REEP5 and PSMB5 signaling. Therefore, circ_0000700 may provide a promising new avenue for the diagnosis and treatment of ESCC.

\section{Materials and Methods}

\section{Cell culture}

The esophageal cancer cell lines KYSE-410, KYSE-150, ECA-109, and TE-1 and the standard esophageal cell line HET-1A were obtained from the Cell Bank of the Chinese Academy of Sciences and incubated in RPMI-1640 medium (Gibco, Grand Island, NY, USA) supplemented with $10 \%$ fetal bovine serum (FBS; Gibco) and $1 \%$ penicillin/ streptomycin (Invitrogen, CA, USA) at $37{ }^{\circ} \mathrm{C}$ in a $5 \%$ $\mathrm{CO}_{2}$ incubator.

\section{Quantitative reverse transcription-polymerase chain reaction ( $q R T-P C R$ )}

Total RNA was extracted from KYSE-410, KYSE-150, ECA-109, TE-1, and HET-1A cells by using TRIzol Reagent (Invitrogen, CA, USA) according to the manufacturer's instructions. For circRNA 
analysis, cDNA was synthesized by using Prime Script ${ }^{\mathrm{TM}}$ RT Master Mix (Takara, Japan), and for miRNA analysis, RNA was reverse transcribed into cDNA using the Mir-X miRNA First-Strand Synthesis Kit (Takara, Japan). The nucleus and cytoplasm were separated with NE-PER Nuclear and Cytoplasmic Extraction Reagents (Thermo Fisher Scientific) following the manufacturer's instructions. qRT-PCR was conducted with SYBR ${ }^{\circledR}$ Premix Ex Taq ${ }^{\mathrm{TM}}$ (Takara) on a CFX96 Touch Deep Well detection system (Bio-Rad, USA) with the following primers: circ_0000700, 5' '-CTGGCTGAACATATCCCTTGGC-3' (forward) and 5'-TCCAAGGGATATGTTCAGCCA-3' (reverse); U6, 5'-CTCGCTTCGGCAGCACA-3' (forward) and 5'-AACGCTTCACGAATTTGCGT-3' (reverse); and GAPDH, 5' -AGA AGGCTGGGGCTCA TTTG-3' (forward) and 5'-AGGGGCCATCCACAGTC TTC-3' (reverse). The entire sequence of the mature miR-1229 miRNA sequence was used as the miRNAspecific $5^{\prime}$ primer, and the $3^{\prime}$ primer for qRT-PCR was the $\mathrm{mRQ} 3^{\prime}$ primer supplied with the kit. GAPDH was used as the reference gene for circ_0000700, and U6 was selected as the internal reference for miR-1229. The relative expression of the aforementioned targets was assessed by the $2^{-\Delta \Delta \mathrm{Ct}}$ method.

\section{Actinomycin D and RNase $R$ treatment}

For actinomycin treatment, $2 \mathrm{mg} / \mathrm{ml}$ actinomycin D (Sigma, USA) was added to the cell culture medium. For RNase R treatment, total RNA (2 $\mu \mathrm{g}$ ) was incubated for $15 \mathrm{~min}$ at $37^{\circ} \mathrm{C}$ with or without $3 \mathrm{U} / \mu \mathrm{g}$ of RNase $\mathrm{R}$ (Epicentre Biotechnologies, China). After treatment with actinomycin D or RNase $\mathrm{R}$, circ_0000700 expression and CHD9 mRNA expression levels were measured by qRT-PCR.

\section{Cell transfection}

To inhibit the expression of circ_0000700 in the ESCC cell lines, three types of siRNA sequences and a negative control (NC) were purchased from GeneChem (Shanghai, China). The sequences were 5'-AACATATCCCTTGGATACTCT-3' for siCirc-1, 5'-ACATATCCCTTGGATACTCTA-3' for siCirc-2, 5'-CCCTTGGATACTCTAAGACCT-3' for siCirc-3, and $5^{\prime}$-AAUUCUCCGAAC GUGUCACGU- $3^{\prime}$ for the NC. For miR-1229 mimic and miR-1229 inhibitor transfection, miR-1229 mimics, the miR-1229 inhibitor, and the corresponding NC were synthesized by RiboBio (Guangzhou, China). The miRNA sequences were as follows: miR-1229 mimics, 5'-GUGGGUAGGGUUUGGGGGAGAGCG-3', and miR-1229 inhibitor, 5' -CGCUCUCCCCCAAACCCUA CCCAC-3'. ECA-109 and TE-1 cells were transfected with Lipofectamine ${ }^{\circledR} 3000$ (Thermo Fisher) according to the manufacturer's instructions, and the knockdown efficiency was measured by qRT-PCR.

\section{Cell viability and proliferation}

Transfected cells were seeded in a 96-well plate at a cell density of $2 \times 10^{3} / \mathrm{ml}$ per well. After culturing for $24,48,72$, or $96 \mathrm{~h}, 10 \mu \mathrm{l}$ of Cell Counting Kit-8 (CCK-8) proliferation solution (Beyotime Biotechnology, China) was added to each well and incubated for $2 \mathrm{~h}$ at $37^{\circ} \mathrm{C}$. The absorbance (OD value) at $450 \mathrm{~nm}$ was then analyzed using a microplate reader.

For the colony formation assay, ECA-109 and TE-1 cells were transfected with $50 \mathrm{nM}$ si_circ 0000700 or related NC, and the transfected cells were plated in six-well plates at a density of 1000 cells per well and incubated in RPMI-1640 medium with 10\% FBS at $37^{\circ} \mathrm{C}$. After 14 days, the visible colonies were fixed with $4 \%$ paraformaldehyde, stained with $0.2 \%$ crystal violet (Sigma), imaged, and then counted.

For the EdU assay, ECA-109 and TE-1 cells transfected with siCircRNA or the related NC were plated in 24-well plates at a density of $5 \times 104$ cells/well and incubated for another $48 \mathrm{~h}$. Following the specified treatment, cells were then incubated in serum-free RPMI-1640 medium containing $10 \mu \mathrm{mol} / \mathrm{L}$ 5-ethynyl-2'-deoxyuridine (EdU, BeyoClick ${ }^{\mathrm{TM}}$ EdU-488, Beyotime Biotechnology, China) for $2 \mathrm{~h}$. The cells were fixed with $4 \%$ paraformaldehyde and then underwent DAPI staining following the manufacturer's protocol. Images were captured using inverted fluorescence microscopy (Leica, Germany), and the green-colored positive cells were further quantified using ImageJ software (National Institutes of Health, Bethesda, MD, USA).

\section{Apoptosis analyses}

Cell apoptosis was assessed on a FACSCanto II flow cytometer (BD Biosciences, San Jose, CA). Cells were transfected, collected and then treated with Annexin V-FITC (Beyotime, China) and propidium iodide (Keygen, China) at room temperature for 10 minutes each following the manufacturer's instructions.

\section{Cell migration}

The migration of ECA-109 and TE-1 cells was evaluated with a Transwell assay carried out using Transwell chambers (Corning, NY). Briefly, $4 \times 10^{4}$ transfected cells/well were suspended in serum-free RPMI 1640 medium and then added into the top compartment, and $500 \mu \mathrm{L}$ of RPMI 1640 medium supplemented with $20 \%$ FBS was placed in the lower chamber. After incubation for $24 \mathrm{~h}$, the cells on the lower filter surface were fixed, stained with $0.5 \%$ crystal violet for 15 minutes at room temperature, and 
digitally imaged in 6 random fields for each chamber using a microscope at $200 \times$ magnification.

\section{Western blot analysis}

Transfected ECA-109 and TE-1 cells were lysed using precooled RIPA lysis buffer (Beyotime, China), and the protein concentration was quantified with the BCA Protein Assay Kit (Beyotime, China). Sodium dodecyl sulfate polyacrylamide gel electrophoresis (SDS-PAGE, 10\%) was employed to separate the proteins, and then the proteins were transferred to polyvinylidene difluoride (PVDF) membranes (Millipore, Billerica, MA, USA). The membranes were blocked with $5 \%$ skim milk in a TBST solution for $1 \mathrm{~h}$ at room temperature and incubated with primary antibodies against Caspase 3 (1:500; ab13847, Abcam), Caspase 9 (1:2,000; ab32539, Abcam), Bax (1:1,000; ab32503, Abcam), Bcl-2 (1:2,000; ab182858, Abcam), E-cadherin (1:500; ab15148, Abcam), Vimentin (1:1,000; \#12826, Cell Signaling Technology, Boston, USA), N-cadherin (1:1,000; \#14215, Cell Signaling Technology, Boston, USA), and GAPDH (1:10,000; R1210-1, HuaBio, Hangzhou, China) at $4{ }^{\circ} \mathrm{C}$ overnight. Then, the membranes were incubated with an appropriate HRP-conjugated secondary antibody at room temperature for $2 \mathrm{~h}$. Finally, the bands were visualized by enhanced chemiluminescence (Solarbio, China), and the intensity of the protein bands was analyzed using ImageJ software.

\section{Dual-luciferase reporter assays}

Circular RNA Interactome (https:// circinteractome.nia.nih.gov/index.html) [14] was used to predict the target miRNAs of circ_0000700. We then used a dual-luciferase reporter assay to confirm whether circ_0000700 contains miR-1229 binding sites. Full-length sequences of circ_0000700 with or without a mutant miR-1229 binding site were cloned into the luciferase vector pGL3 (Promega, Madison, USA). ECA-109 and TE-1 cells were cotransfected with the mutant or wild-type circ_0000700 luciferase vectors and miR-1229 mimics or the related NC with Lipofectamine ${ }^{\circledR} 3000$ (Thermo Fisher). After $24 \mathrm{~h}$ of transfection, luciferase activity was estimated with a dual-luciferase reporter assay kit (RG027, Beyotime, China).

\section{RNA immunoprecipitation (RIP)}

Experiments were performed based on the protocol of an EZMagna RIP kit (Merck, Darmstadt, Germany). In brief, ECA-109 and TE-1 cell lysates were prepared with RIP lysis buffer, and the supernatant was incubated with magnetic beads that were preconjugated with an anti-IgG or anti-Argonaute 2 (AGO2) antibody for $6 \mathrm{~h}$ at $4{ }^{\circ} \mathrm{C}$. Subsequently, the magnetic beads were washed and incubated with protease $\mathrm{K}$ to digest the proteins. Finally, the levels of circ_0000700 and miRNA-1229 were detected by qRT-PCR.

\section{Bioinformatic analysis}

TargetScan (http://www.targetscan.org/ vert_72/) [15], miRPathDB 2.0 (https://mpd.bioinf. uni-sb.de/overview.html) [16], and miRTarBase (http:// mirtarbase.mbc.nctu.edu.tw/php/index. php) [17] were employed to predict the target genes of miRNA-1229. In addition, the intersection of the aforementioned databases was analyzed through Venny 2.1 (https://bioinfogp.cnb.csic.Es/tools/ venny/index.html). Then, a circRNA-miRNA-target regulatory network was visualized with Cytoscape software (v3.7.2, https://cytoscape.org/). To further investigate the biological function of the intersected targets, Kyoto Encyclopedia of Genes and Genomes (KEGG) pathway and Gene Ontology (GO) enrichment analyses were performed using the Metascape (http://metascape.org/gp/index.html\#/ main/step1) database [18]. Additionally, overall survival (OS) related to the intersected targets was evaluated with Kaplan-Meier plotter (http:// kmplot. com/analysis/index.php?p=service) [19]. Finally, the mRNA expression levels of selected intersected targets were analyzed with the UALCAN cancer database (http://ualcan.path.uab.edu/analysis.html) [20].

\section{Statistical analysis}

All data are expressed as the mean \pm standard deviation (SD), and statistical analyses were performed using SPSS 22.0 software (IBM, Chicago, USA). Statistical differences between two groups were evaluated by Student's t-test, and one-way analysis of variance (ANOVA) was used for comparison of three or more groups. $P<0.05$ was considered statistically significant.

\section{Results}

\section{Biological characteristics of hsa_circ_0000700 in ESCC cells}

According to the circBase database, hsa_circ_0000700 is generated from the q12.2 region of chromosome 16, with a length of 82 bases, and derived from CHD9 mRNA (Figure 1A). To explore the expression levels of circ_0000700 in ESCC cells, we used qRT-PCR to investigate circ_0000700 expression in a normal esophageal epithelial cell line (HET-1A) and ESCC cell lines (KYSE-410, KYSE-150, ECA-109, and TE-1), revealing that circ_0000700 expression in ESCC cells was remarkably increased (Figure 1B). We used ECA-109 and TE-1 cells as the experimental cell lines due to their high expression of circ_0000700. 
Additionally, RNase R exonuclease and actinomycin experiments were employed to confirm the circular nature of circ_0000700 in ECA-109 and TE-1 cells, respectively. The results suggested that circ_0000700 was resistant to the RNase $\mathrm{R}$ exonuclease and actinomycin, whereas linear CHD9 mRNA was not expressed in ECA-109 or TE-1 cells (Figure 1C and 1D). Moreover, a nuclear and cytoplasmic extraction experiment was conducted to investigate the subcellular localization of circ_0000700 in ECA-109 and TE-1 cells, and it was observed that most circ_0000700 was mainly concentrated in the cytoplasm of ECA-109 and TE-1 cells (Figure 1E). Taken together, these studies suggest that circ_0000700 is a stable circRNA that could be associated with the occurrence and development of ESCC and participate in posttranscriptional modification.

\section{Downregulation of circ_0000700 expression inhibits progression in ESCC cells}

To further evaluate the role of circ_0000700 in ESCC, circ_0000700 expression in ECA-109 cells was knocked down through transfection of siCirc-1/2/3. The knockdown efficiency was confirmed using qRT-PCR, and the results indicated that the siCirc-3 vector significantly decreased the endogenous circ_0000700 expression level (Figure 2A). Subsequently, a CCK-8 assay was employed to assess the effect of circ_0000700 on cell viability. The results showed that the viability of ECA-109 cells decreased observably after knocking down circ_0000700 expression (Figure 2B). With the depletion of circ_0000700, TE-1 cells displayed similar tendencies

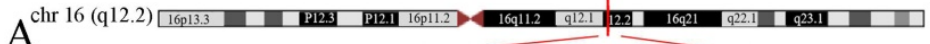
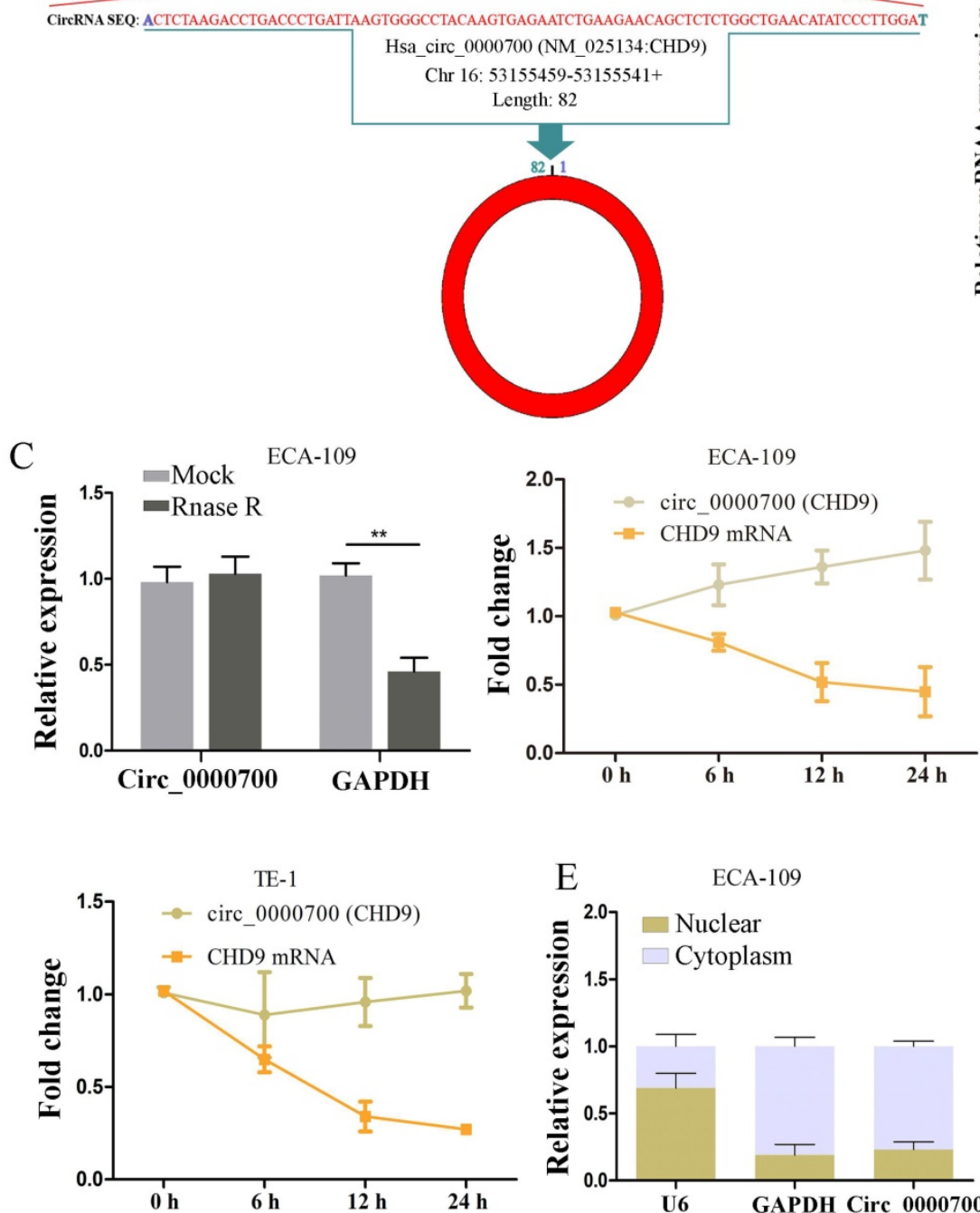

$\mathrm{E}$

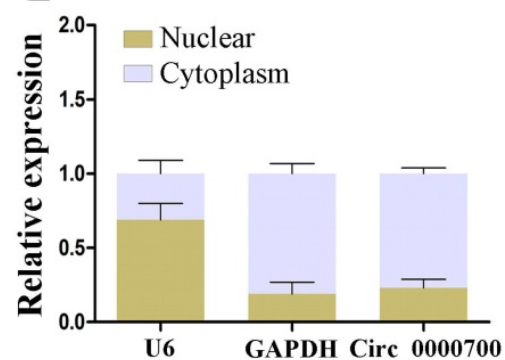

$\mathrm{B}$
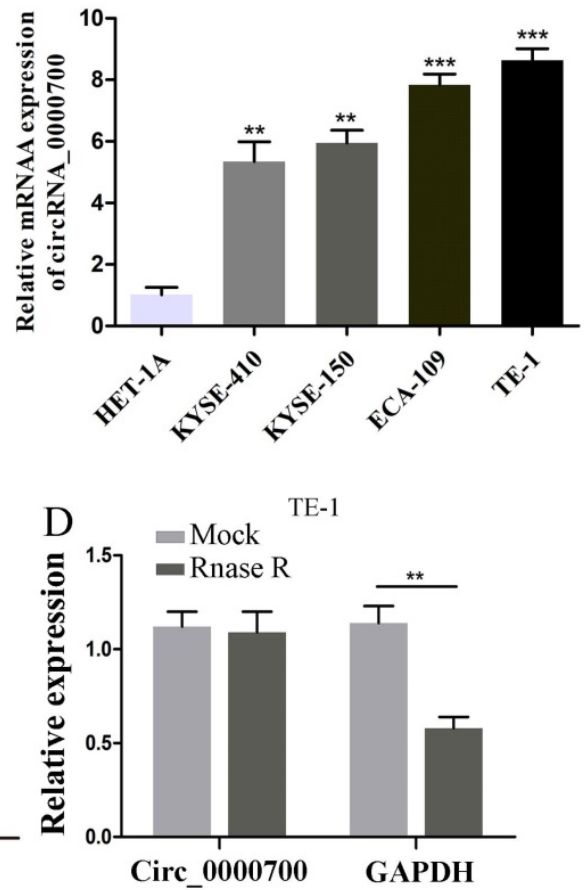

TE-1

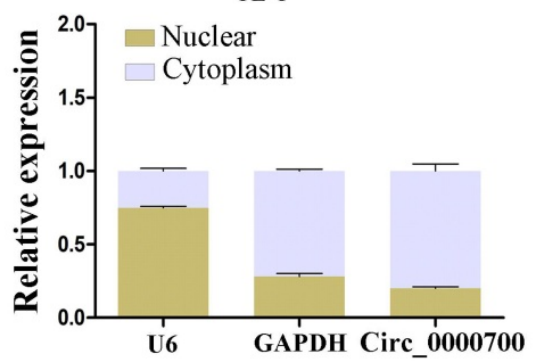

Figure 1. Characterization of circ_ $\mathbf{0 0 0 0 7 0 0 ~ i n ~ e s o p h a g e a l ~ c a r c i n o m a ~ c e l l ~ l i n e s . ~ ( A ) ~ S c h e m a t i c ~ i l l u s t r a t i o n ~ s h o w i n g ~ t h e ~ c i r c u l a r i z a t i o n ~ o f ~ c i r c ~} 0000700$ by back splicing from chromosomal region 16q12.2. (B) The expression of circ 0000700 in esophageal carcinoma cell lines was detected by qRT-PCR. (C, D) The RNase R and actinomycin assays were used to evaluate the abundance of circ_0000700 and CHD9 mRNA in ECA-109 and TE-1 cells, respectively. (E) The expression levels of U6, GAPDH, circ_0000700 in cytoplasmic and nuclear fractions of ECA-109 and TE- 1 cells were measured by qRT-PCR. ${ }^{*} P<0.05,{ }^{* * *} P<0.01$ and ${ }^{* * * *} P<0.001$. 
for knockdown efficiency and cell viability (Figure 2C and 2D). In addition, as shown in Figure 2E and 3A, compared with NC treatment, siRNA treatment of ECA-109 and TE-1 cells significantly decreased the number of cell colonies and EdU incorporation. Then, flow cytometric analyses were performed with ECA-109 and TE-1 cells. As illustrated in Figure 3B, cell apoptosis was apparently increased in the two cell lines after circ_0000700 silencing. As shown in Figure $3 \mathrm{C}$, the number of migrated cells was significantly
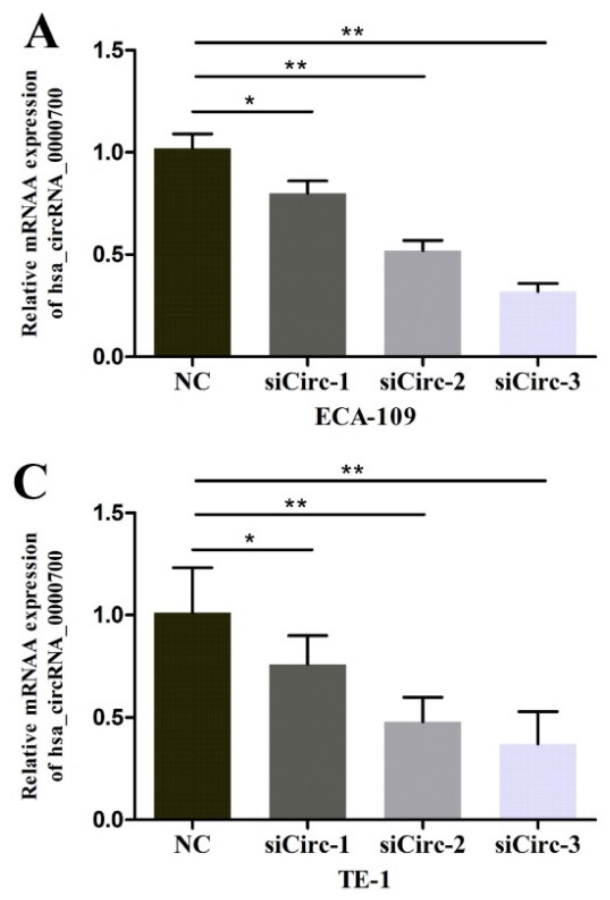

$\mathbf{E}$

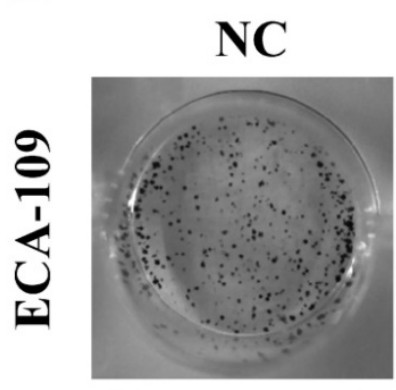
siCirc-3
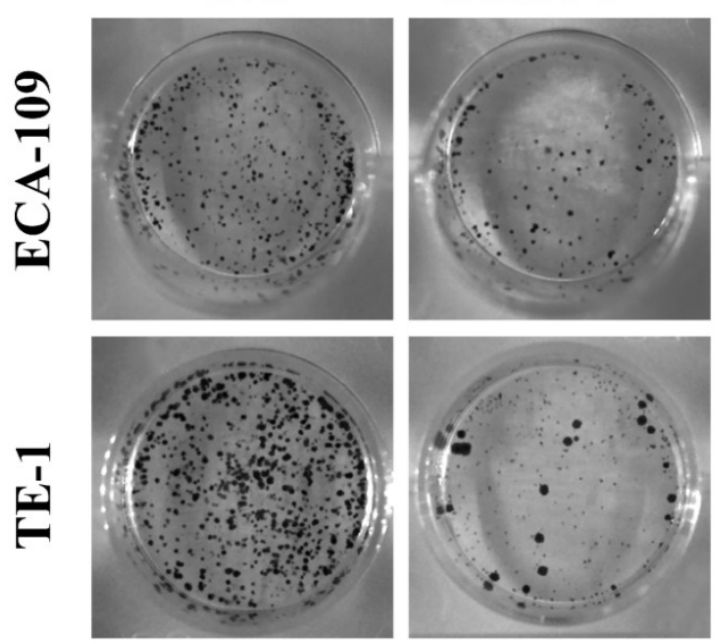

decreased in the circ_0000700-knockdown group compared with the NC group. In addition, by Western blot analysis of ESCC cells, compared with the NC group, the knockdown group showed downregulated circ_0000700 expression; significantly elevated Caspase-3, Caspase-9, Bax, and E-cadherin protein expression levels; and reduced Bcl-2, vimentin, and $\mathrm{N}$-cadherin protein expression levels (Figure 3D). The above results indicated that circ_0000700 exerted a negative regulatory effect on ESCC cells.
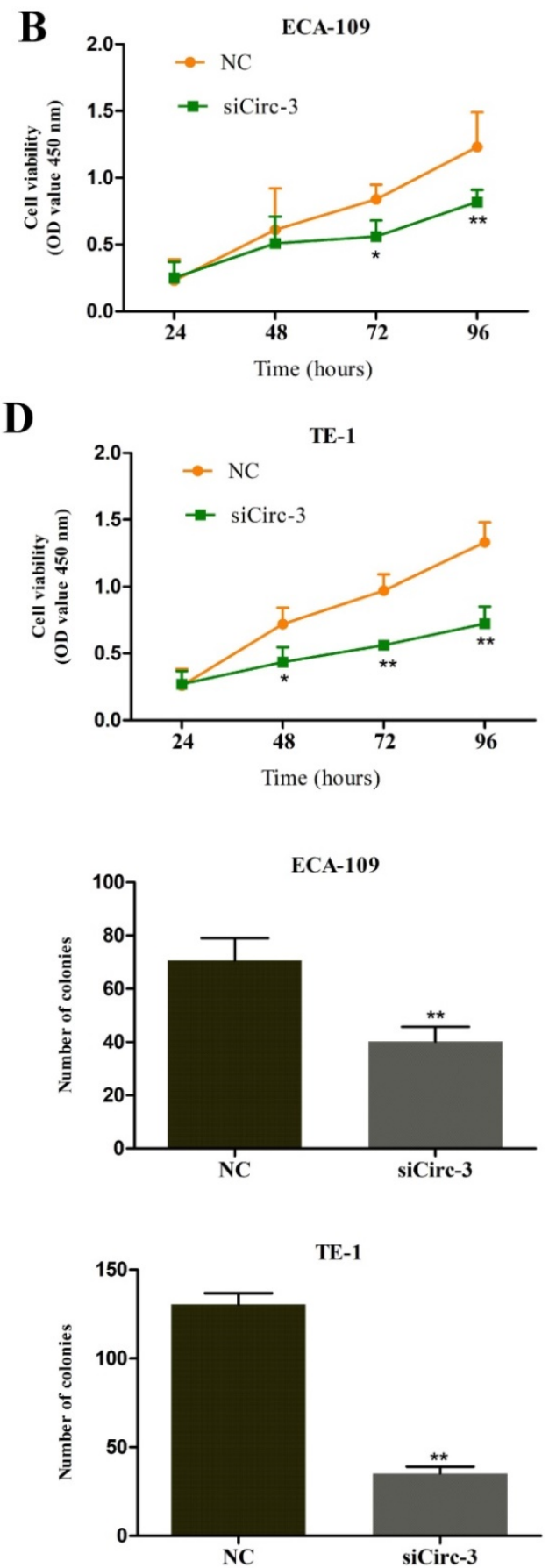

Figure 2. Downregulation of circ 0000700 inhibits the proliferation of ESCC cells in vitro. (A) The knockdown efficiency of circ 0000700 by siRNA interference in ECA-109 cells. (B) The effect of the silencing of circ_0000700 on cell viability of ECA-109 cells in vitro using the CCK-8 assay. (C) The knockdown efficiency of circ_0000700 by siRNA interference in TE-1 cells. (D) The effect of the silencing of circ_0000700 on cell viability of TE-1 cells in vitro using the CCK-8 assay. (E) Down-regulation of circ_0000700 inhibits the colony-forming ability of ECA- 109 and TE-1 cells. ${ }^{*} P<0.05,{ }^{*} * 0001$. 

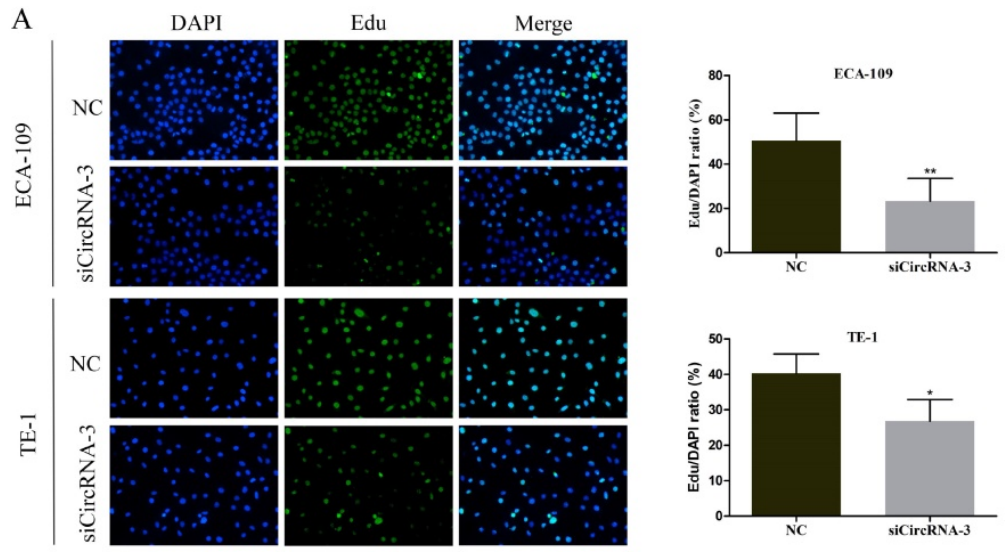

B
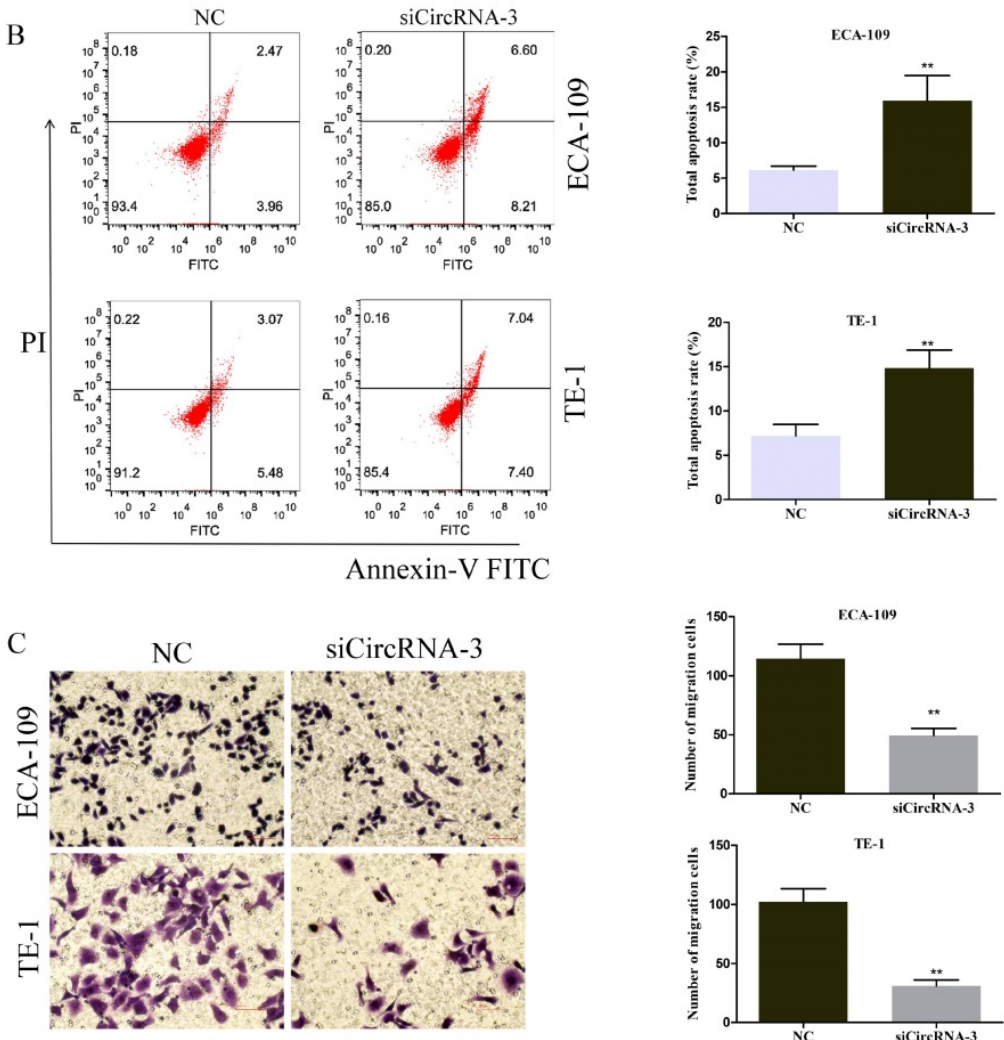

D
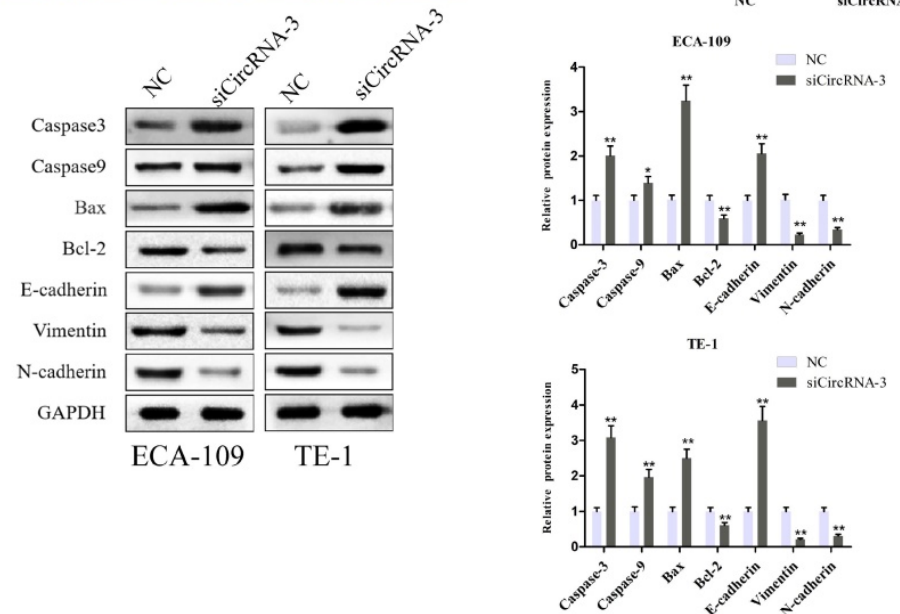

Figure 3. Downregulation of circ_0000700 promotes apoptosis and suppresses proliferation, migration capacities of ESCC cells in vitro. (A) EdU assay of ECA- 109 and TE-1 cells transfected with negative control (NC) or circ 0000700 siRNA was performed to evaluate cell proliferation ability. (B) Flow cytometry apoptosis analysis of ECA-109 and TE-1 cells transfected with NC or circ 0000700 siRNA. (C) Cell migration assays were performed in ECA-109 and TE-1 cells using Transwell chambers. (D) Western blot showed that the silencing of circ_0000700 can promote the expression of Caspase-3, Caspase-9, Bax, and E-cadherin while restraining the protein levels of Bcl-2, vimentin, and $\mathrm{N}$-cadherin. ${ }^{*} \mathrm{P}<0.05,{ }^{* * P}<0.01$. 
A WT hsa_circ_0000700 5'...UAAGUGGGCCUACAAGUGAGAAU....3'

hsa_miR-1229 GACACCCUCCCGUCAC -- CACUCUC

MUT hsa circ $0000700 \quad 5^{\prime}$...UAAGUGGGCCUACAACACUCUAU...3'
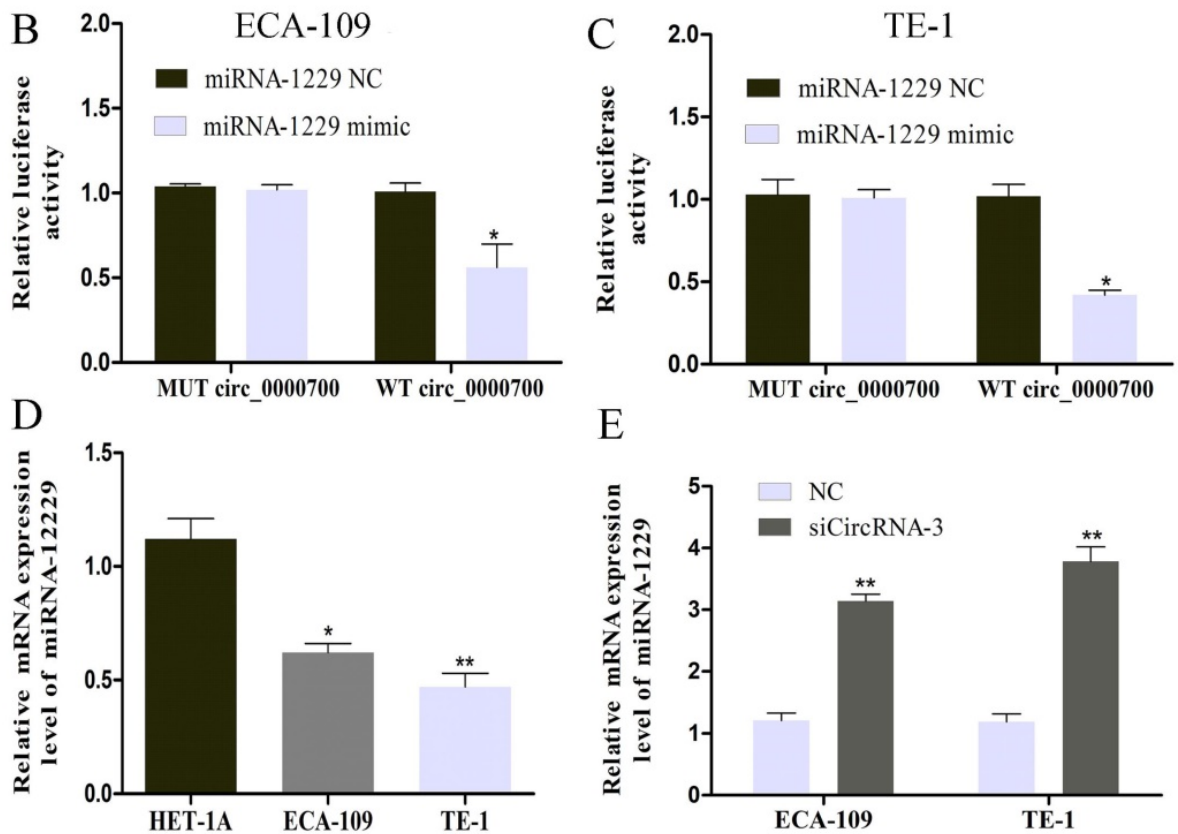

$\mathrm{E}$
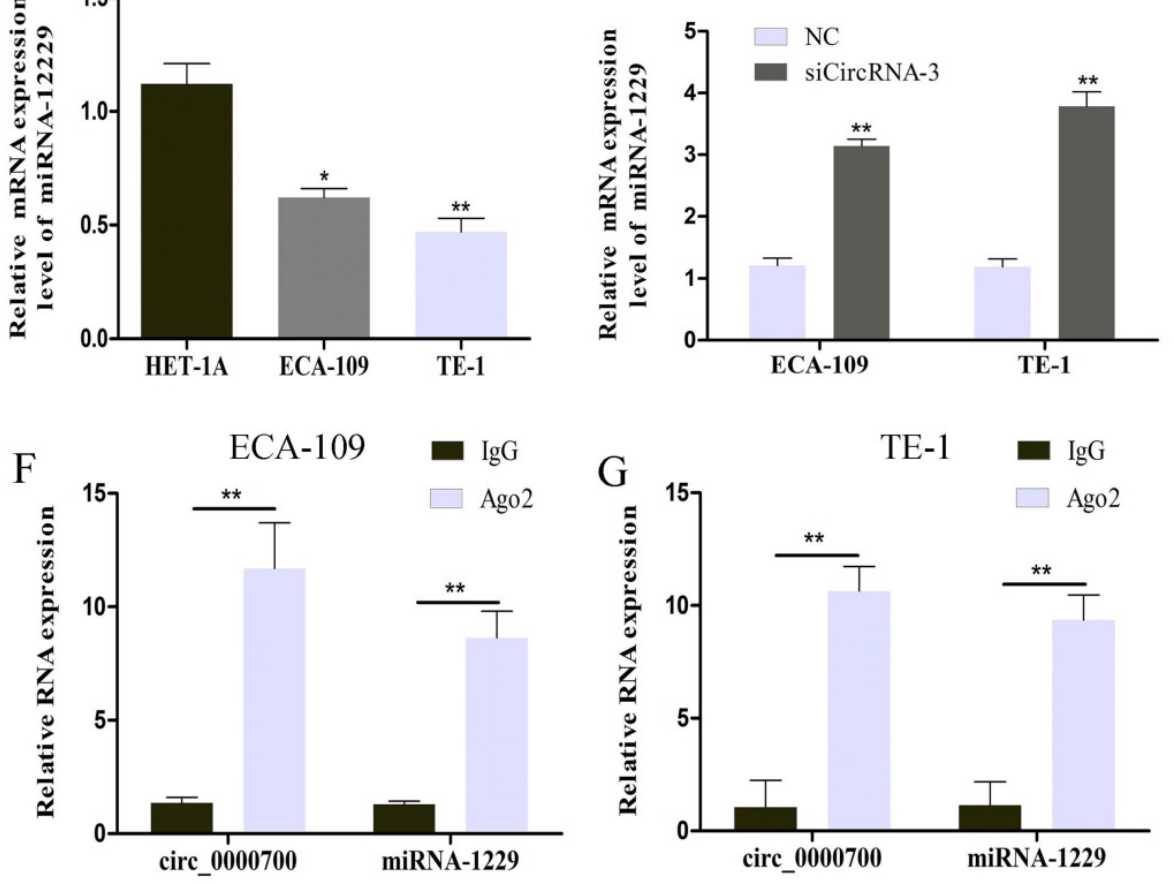

Figure 4. circ_0000700 targets miR-1229 as a miRNA sponge. (A) The possible binding sites of miR-1229 within circ_0000700. The luciferase activity decreased after cotransfection with miR-1229 mimics and wild-type circ_0000700 in ECA-109 (B) and TE-1 (C) cell lines. (D) The expression of miR-1229 in ECA-109 and TE-1 cells. (E) The miR-1229 level was increased in the ESCC ECA-109 and TE-1 cells transfected with NC or circ 0000700 siRNA. (F and G) RIP and qRT-PCR assays measure the differences of circ_0000700 and miR-1229 levels between the Ago2 and the IgG immunoprecipitation. ${ }^{*} P<0.05,{ }^{* *} P<0.01$.

\section{Circ_0000700 is a sponge of $\mathrm{miR}-1229$}

The cytoplasmic distribution of circ_0000700 indicated that it might function by sponging miRNAs. We then used the Circular RNA Interactome database to predict the potential miRNAs targeted by circ_0000700. As shown in Figure 4A, bioinformatic analysis results identified possible binding sites of circ_0000700 and miR-1229. To further investigate the relationship between circ_0000700 and miR-1229, a luciferase assay was conducted with ECA-109 and TE- 1 cells, and the results showed that overexpression of miR-1229 led to tremendous attenuation of luciferase activity in the wild-type (WT) circ_0000700 group (Figure $4 \mathrm{~B}$ and $4 \mathrm{C}$ ). To explore the role of
miR-1229 in ESCC, the miR-1229 expression level was also measured. As shown in Figure 4D, the expression level of miR-1229 was remarkably downregulated in ECA-109 and TE-1 cells in comparison to HET-1A cells. In addition, a qRT-PCR experiment also revealed that the miR-1229 expression level in ECA-109 and TE-1 cells was significantly increased through knockdown of circ_0000700 expression (Figure 4E). Subsequently, we also performed a RIP assay for Ago2 and found higher levels of circ_0000700 and miR-1229 in the Ago2 group than in the IgG group for ECA-109 and TE-1 cells (Figure 4F and $4 \mathrm{G}$ ). Taken together, these findings indicated that miR-1229 might be a significant downstream target of circ_0000700 in ESCC. 


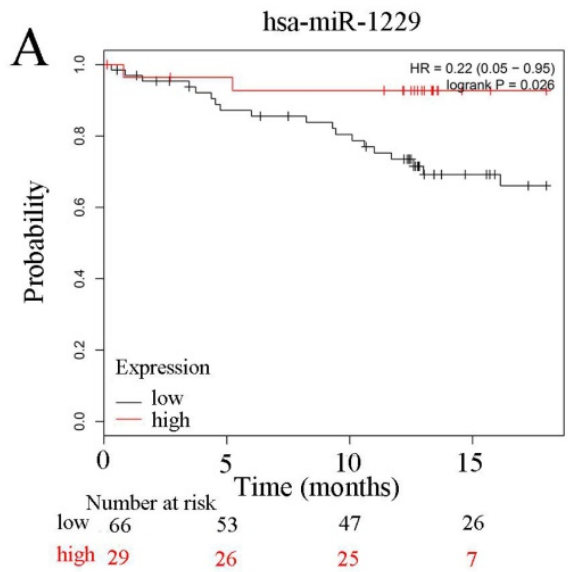

$\mathrm{B}$

$\mathrm{C}$
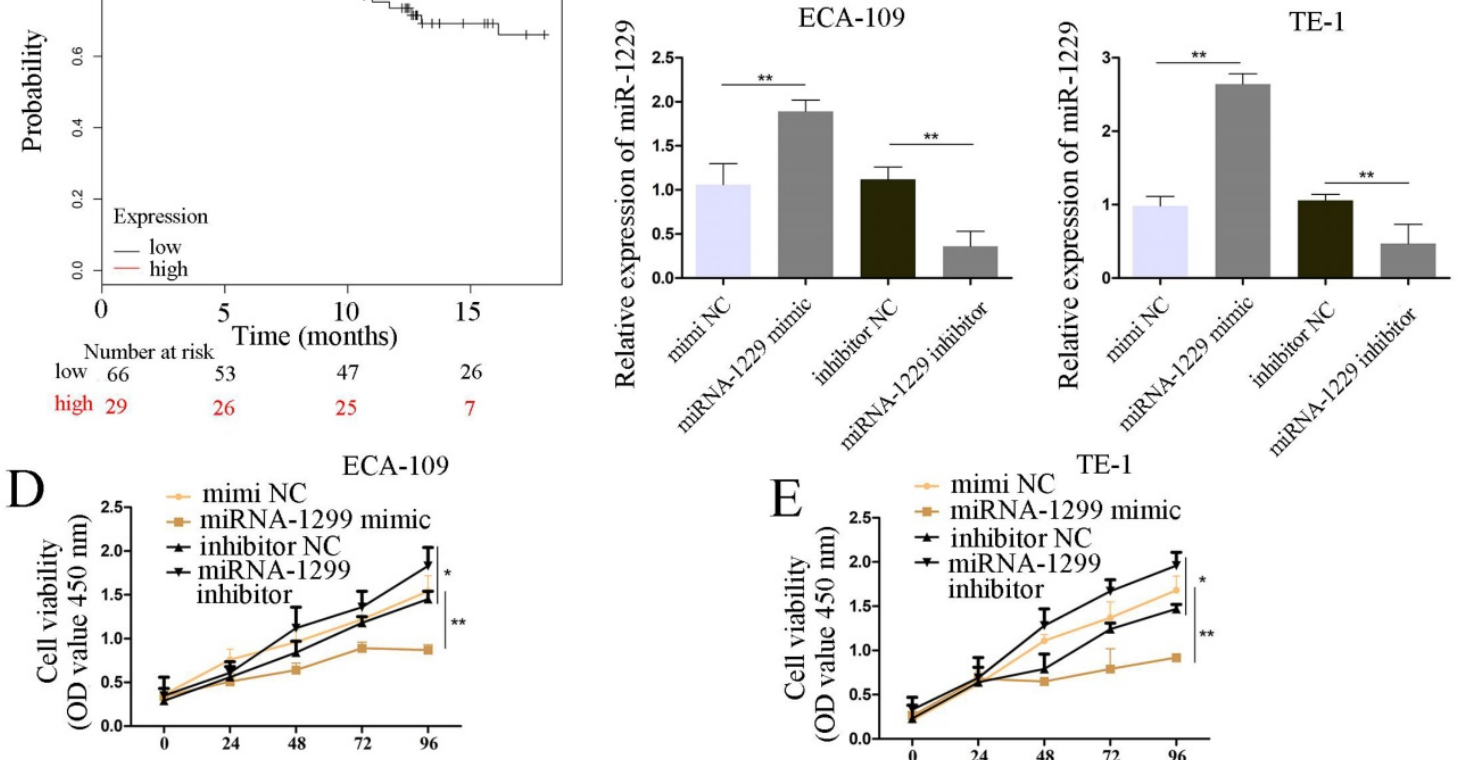

$\mathrm{F}$
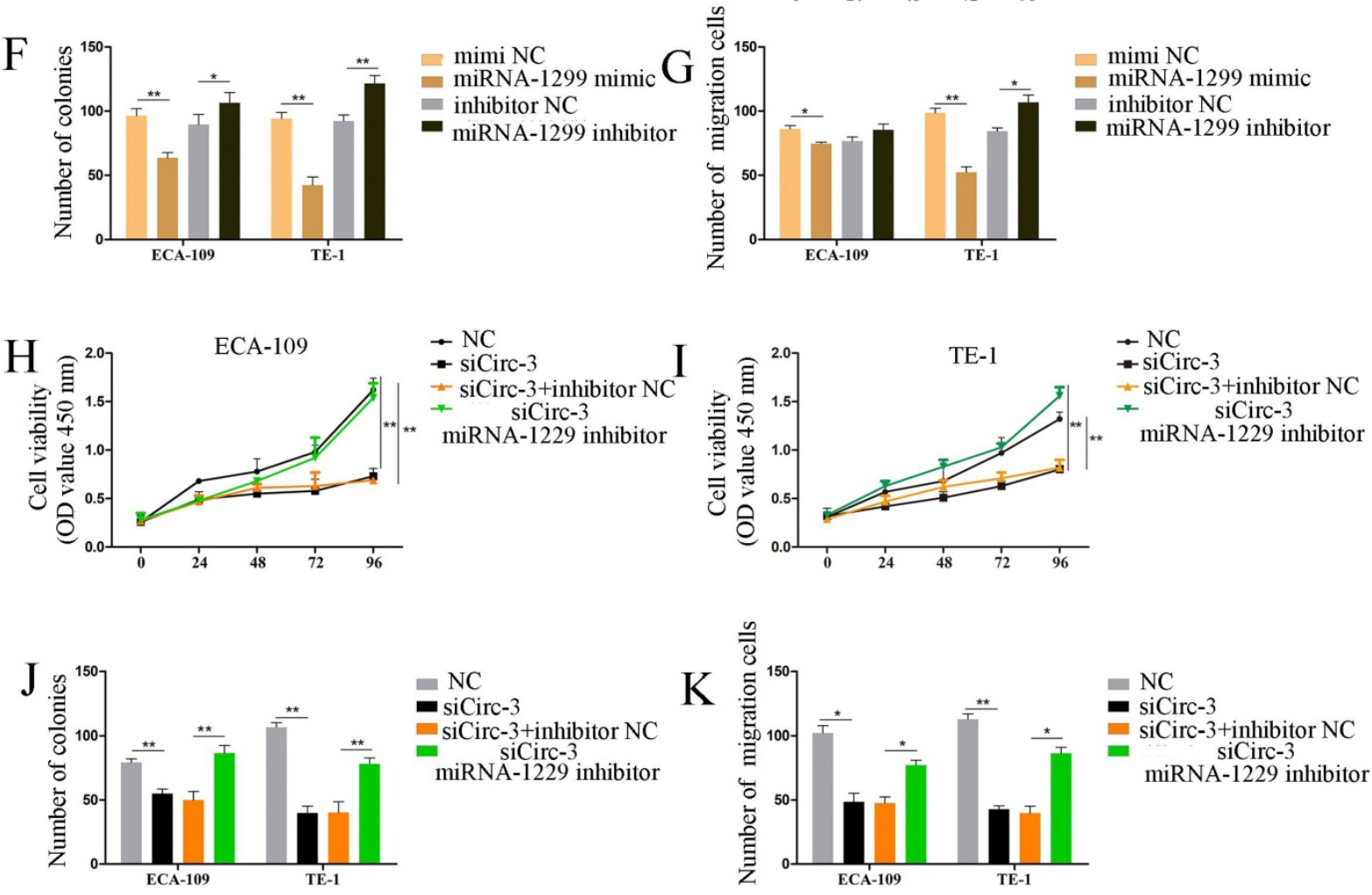

Figure 5. miR-1229 acts as a tumor suppressor gene in ECA-109 and TE-1 cells, and the silencing of miR-1229 effectively reverses circ_0000700 siRNA induced inhibition of proliferation and migration. (A) The overall survival curve of ESCC patients with low and high miR-1229 expression. (B and C) The expression level of miR-1229 in ECA-109 and TE-1 cells transfected with miR-1229 mimics and miR-1229 inhibitor. (D, E, F, and G) Cell proliferation, colony formation, and cell migration assays were determined in ECA-109 and TE-1 cells following transfection with miR-1229 inhibitor, miR-1229-NC or miR-1229 mimics. (H, I, J, and K) ECA-109 and TE-1 cells transfected with circ $0000700-\mathrm{NC}$, miR-1229 inhibitor NC, miR-1229 inhibitor, or circ 0000700 siRNA alone or simultaneously. Then, the cell proliferation and migration were, respectively, assessed by CCK-8 $(\mathrm{H}$ and $\mathrm{I})$, clone assay $(\mathrm{J})$, and the transwell migration $(\mathrm{K})$ assay. ${ }^{*} \mathrm{P}<0.05,{ }^{* *} \mathrm{P}<0.01$.

\section{miR-1229 inhibited ESCC cell proliferation and migration}

To investigate the clinical significance of miR-1229 in ESCC, we divided ESCC patients into high and low expression groups by using Kaplan-Meier plotter (http://kmplot.com/). As demonstrated in Figure 5A, a low miR-1229 expression level was associated with unfavorable overall survival (OS, hazard ratio $[\mathrm{HR}]=0.22 ; 95 \% \mathrm{CI}$ : 
0.05-0.95, $P=0.026)$. Accordingly, to elucidate the biological effect of miR-1229 in ESCC, miR-1229 mimics or a miR-1229 inhibitor was transfected into ECA-109 and TE-1 cells, and then qRT-PCR analysis was conducted to evaluate the transfection efficiency, suggesting that we successfully overexpressed and knocked down miR-1229, respectively, in the two cell lines (Figure 5B and 5C). Compared with the NC group, the miR-1229 mimic group showed reduced cell viability, colony numbers and cell migration (Figure 5D-5G). Furthermore, inhibition of miR-1229 expression had the opposite effects (Figure 5D-5G). To further validate the results for the circ_0000700/ miR-1229 axis, we performed rescue experiments in vitro. As shown in Figure $5 \mathrm{H}-5 \mathrm{~K}$, the miR-1229 inhibitor partly reversed the effects of circ_0000700- specific siRNA on the proliferation and migration of ESCC cells. In summary, these data reveal that the circ_0000700/miR-1229 axis is involved in ESCC progression.

\section{Bioinformatic analysis of candidate targets of miR-1229}

In this study, we also predicted the candidate target genes of miR-1229 with the TargetScan, miRPathDB, and miRTarBase databases (Figure 6A). Next, we intersected the results from the aforementioned databases and found that 124 predicted target genes, including DCTD, ALS2, REEP5, NXPE3, CHD4, and PSMB5, could be directly targets of miR-1229. Then, the hsa_circ_0000700/hsa miR_1229/mRNA axis interaction network was
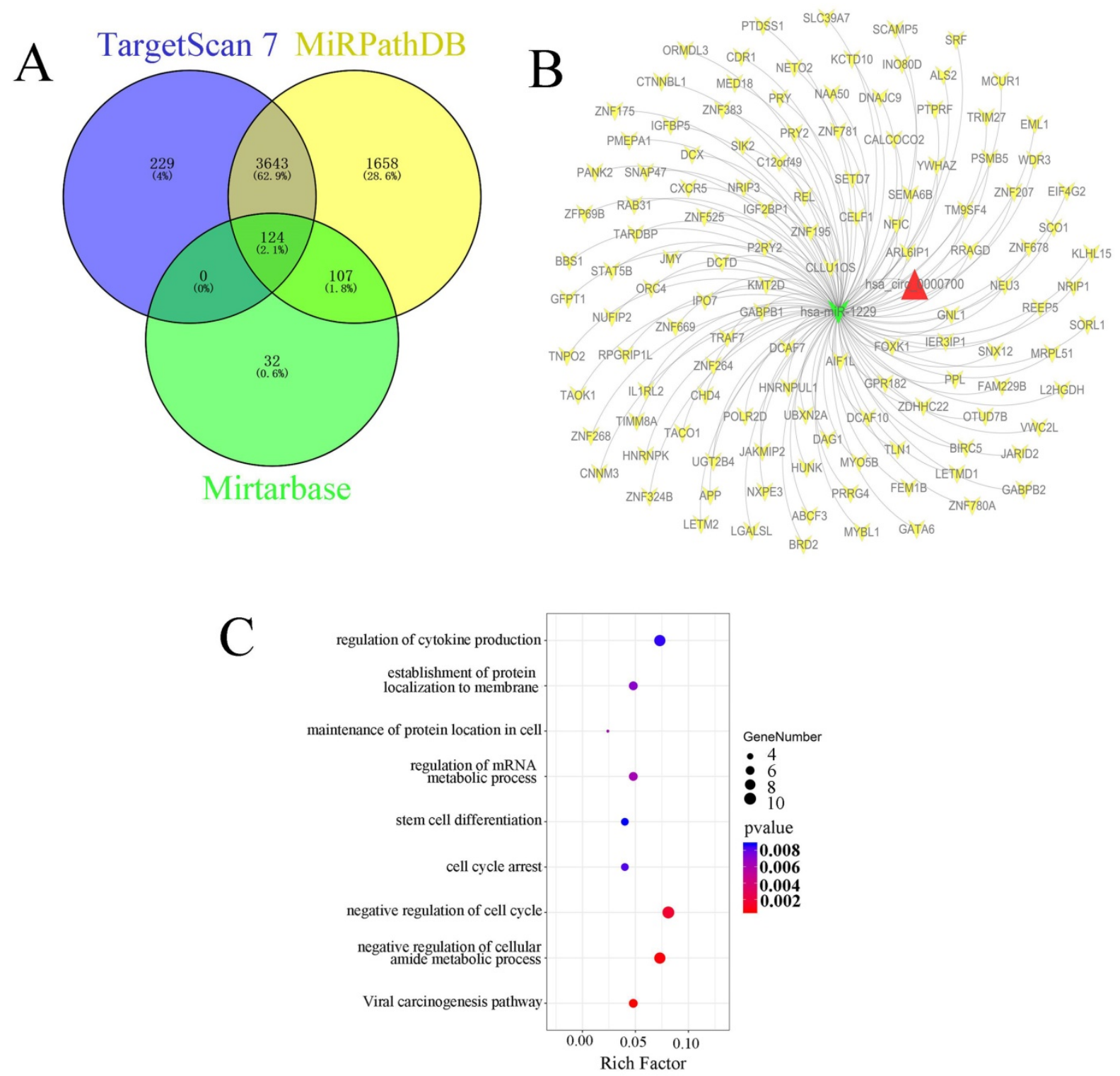

Figure 6. Identification and functional enrichment analysis of predicted targets of miR-1229 (A) Venn diagram of predicted target genes from the intersection of TargetScan, miRPathDB 2.0, and miRTarBase database. (B) The ceRNA network of circ_0000700-miR-1229-mRNA. The red triangle indicates circ_0000700, green "V" indicates miR-1229, and yellow "V" indicates the mRNAs. (C) The KEGG pathway and GO enrichment analysis of predicted target genes. 
constructed (Figure 6B). To determine the biological significance of target genes, KEGG pathway and GO enrichment analyses were performed. We observed that the target genes were significantly associated with the viral carcinogenesis pathway, regulation of the cellular amide metabolic process, negative regulation of the cell cycle, and regulation of cytokine production (Figure 6C). These results suggested that the predicted genes exhibited a close relationship with the development and progression of ESCC. Moreover, the clinical significance of predicted target gene expression in ESCC was further assessed according to Kaplan-Meier survival analysis. We found that low expression of each of 26 target genes including CELF1, CHD4, DCAF10, DNAJC9, FOXK1, GATA6, GNL1, GPR182, HNRNPK, HNRNPUL1, IGF2BP1, JMY, L2HGDH, NEU3, NRIP3, ORMDL3, OTUD7B, POLR2D, SCO1, SIK2, SLC39A7, TIMM8A, TNPO2, UBXN2A, WDR3, and ZFP69B was significantly associated with poor OS (Figure S1). In addition, Kaplan-Meier analysis also revealed that high expression of PRRG4 (HR=2.75; 95\% CI: 1.09-6.92, $P=0.025)$, REEP5 (HR=3.23; 95\% CI: 1.39-7.52, $P=0.0042)$, and PSMB5 (HR=3.09; 95\% CI: 1.35-7.11, $P=0.0052)$ was associated with lower survival rates (Figure 7A-7C). In addition, the predicted binding sites of hsa_miR_1229 within PRRG4, REEP5, and PSMB5 are shown in Figure 7D. Finally, we found that the expression of PRRG4, REEP5, and PSMB5 in primary tumor tissues was significantly higher than that in normal tissues (Figure 7E-7G). Moreover, as shown in Figure 7H-7J, the expression levels of PRRG4, REEP5, and PSMB5 were significantly associated with TNM stage to a certain extent. Therefore, we speculated that downregulation of miR-1229 expression promoted proliferation and migration in ESCC by targeting PRRG4, REEP5, and PSMB5.

\section{Discussion}

Esophageal squamous cell cancer (ESCC) is a malignant tumor with high morbidity and mortality, and clinical outcomes are not satisfactory due to the lack of early diagnosis and limitations of conventional treatment strategies. Therefore, it is of clinical significance to further investigate novel therapeutic targets and sensitive diagnostic biomarkers in ESCC to improve the poor patient prognosis. Increasing evidence indicates that circRNAs, characterized by covalently closed-loop structures, are closely associated with the occurrence and development of multiple cancers, including breast cancer [21], gastric cancer [22], and ESCC [23], making them potential therapeutic targets.

In this study, a novel differentially expressed
circRNA named hsa_circ_0000700 was identified based on the research of Shi et al [13], and the expression of this circRNA was remarkably upregulated in ESCC cell lines compared with HET$1 \mathrm{~A}$ cells. Subsequently, the biological characteristics of circ_0000700 were evaluated in ECA-109 and TE-1 cells, and the results showed that circ_0000700 was reasonably static and highly expressed in the cytoplasm of ESCC cells, which indicates that the underlying molecular mechanism of circ_0000700 is possible posttranscriptional regulation. The above results suggested that circ_0000700 might play an essential role in the biological events of ESCC. Next, we knocked down the expression of circ_0000700 in ECA-109 and TE-1 cells and assessed the effects on cell viability, proliferation, apoptosis, and migration. Functional experiments confirmed that knocking down circ_0000700 expression significantly inhibited the proliferation and migration of ESCC cells, suggesting that circ_0000700 serves as an oncogene in ESCC progression.

Numerous studies have shown that circRNAs may serve as oncogenic or tumor-suppressive genes by regulating epithelial-mesenchymal transition (EMT). For example, hsa_circ_0006948 can induce EMT to promote migration and invasion by sponging miR-490-3p in ESCC [24]. He et al. found that overexpression of circVRK1 depressed cell proliferation, migration, and EMT and reversed radioresistance through activation of PTEN mediated by sponging miR-624-3p and inhibiting the PI3K/AKT signaling pathway in ESCC progression [25]. Moreover, we found that circ_0000700 not only promoted apoptosis in ESCC cell lines in vitro but also had a regulatory role in EMT in ESCC cells. However, the potential mechanism by which circ_0000700 participates in the EMT process needs further experimental verification.

Currently, it has been reported that circRNAs can act as miRNA sponges to participate in posttranscriptional regulation in multiple types of tumors [26]. For instance, the circRNA SMARCA5 is able to suppress the proliferation of cervical cancer cells by modulating miR-432 [27]. circVAPA was found to promote migration and invasion in breast cancer by binding miR-130a-5p in the cytoplasm [28]. Overexpressed circLARP4 inhibits cell proliferation and migration but promotes cell apoptosis by sponging miR-1323, which positively regulates PTEN expression in ESCC [29]. Using bioinformatic analysis, we predicted miR-1229 as a potential target of circ_0000700 and further confirmed this result in dual-luciferase reporter and RIP assays, as well as functional enrichment analysis. Our data suggested that miR-1229 overexpression significantly inhibited 
ECA-109A and TE-1 cell proliferation and migration and that miR-1229 deficiency in circ_0000700-deficient cells substantially abolished circ_0000700circKDM4C-mediated biological effects. Previously, miR-1229 was reported to participate in the viability and apoptosis of FLT3-ITD-positive acute myeloid leukemia cells [30]. In renal cell carcinoma, circ-EGLN3 knockdown suppresses proliferation and aggressiveness by targeting miR-1229 [31]. Additionally, Meng L et al. reported that miR-1229 promotes starvation- or rapamycin-induced autophagy in ESCC cells by directly binding to epidermal growth factor receptor (EGFR) [32]. In addition, the GO and KEGG enrichment analyses of targets of miR-1229 suggested that miR-1229 might affect the viral carcinogenesis pathway and regulation of the cell cycle. According to the above results, circ_0000700 knockdown could decrease the degradation of miR-1229 to restrain the growth of ESCC.

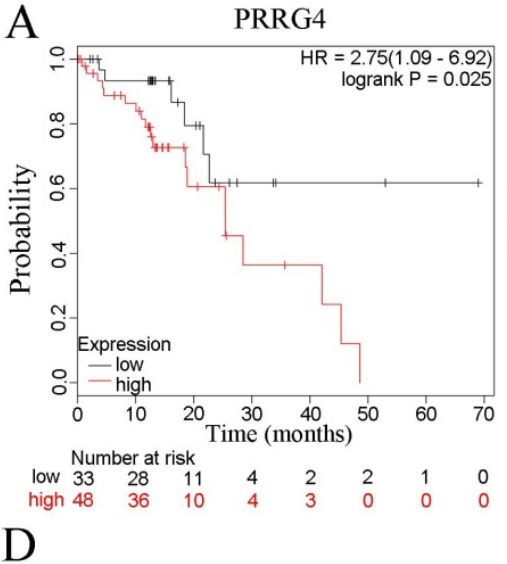

Position 1989-1995 of PSMB5 3' UTR 5' ...GAAAUGGUUAGCUGCGUGAGAAG... Position 1179-1185 of REEP5 3' UTR 5' ...AUGCAAGUGAACAAAGUGAGAAA... Position 2719-2725 of PRRG4 3' UTR 5' ...CUAGCUUCAGAACCUGUGAGAAC... hsa-miR-1229 3' GAC

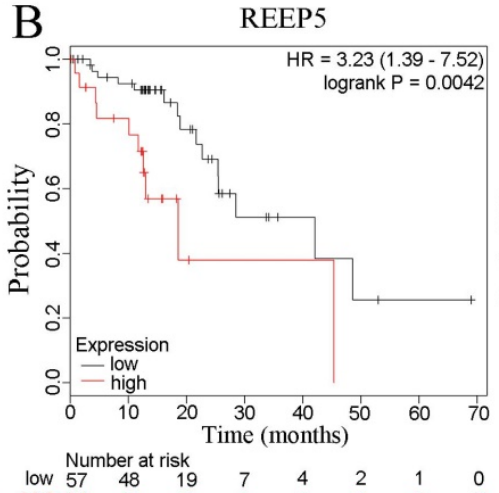

| | | || |

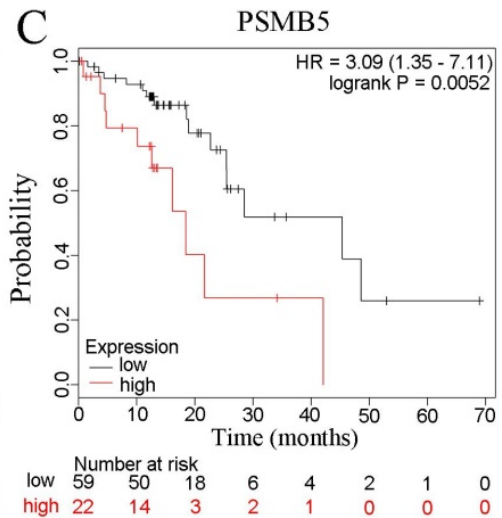

E Expresion of PRRG4 in ESCA based on sample types

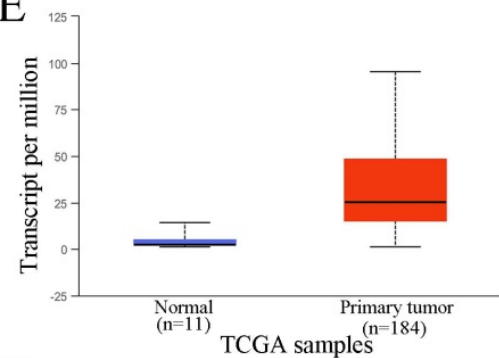

$\mathrm{H}$

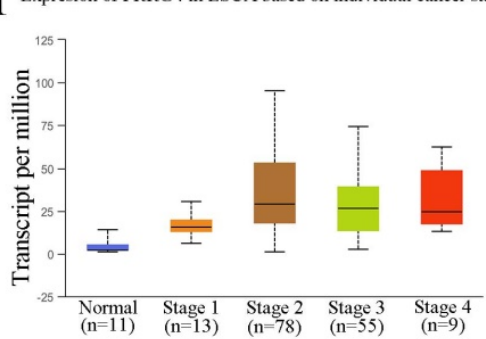

TCGA samples
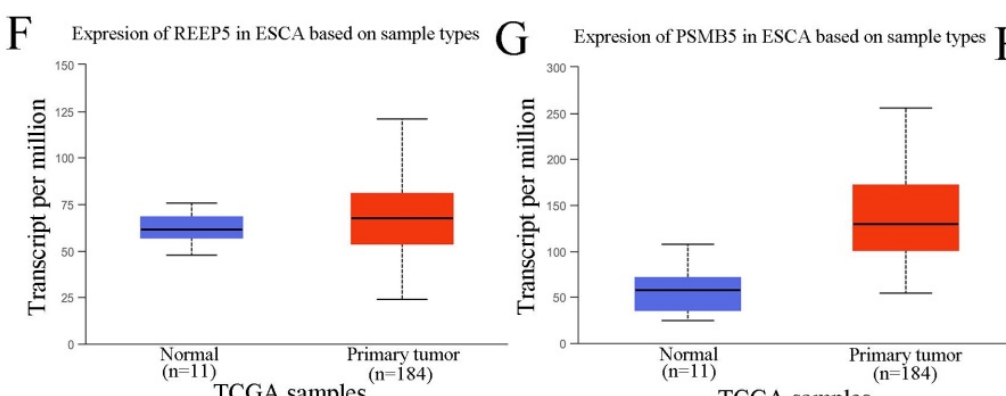

I

Expresion of REEP5 in ESCA based on individual cancer J Expresion of PSMB5 in ESCA based on individual cancer
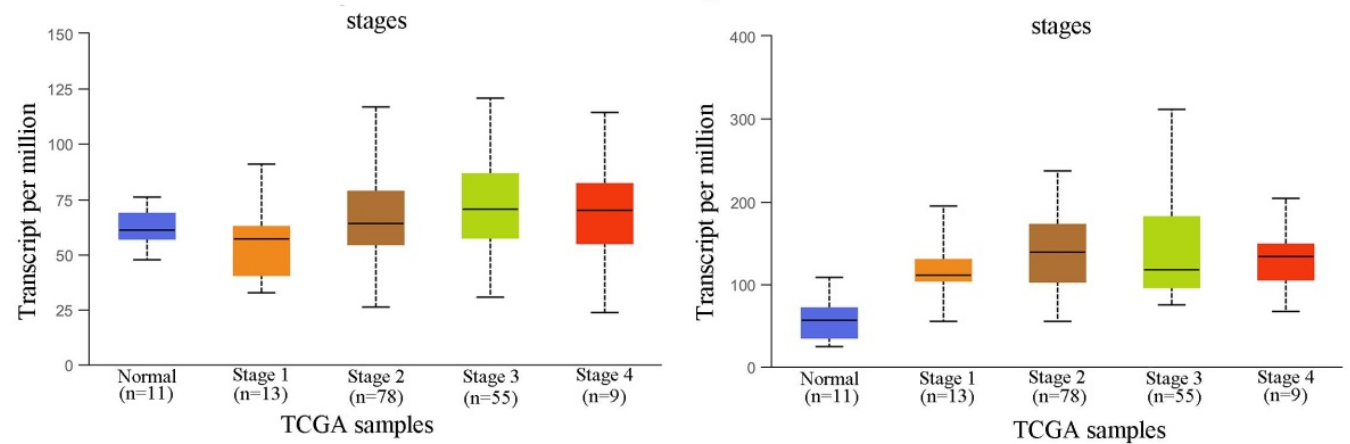

Figure 7. The relationship of PRRG4, REEP5, and PSMB5 with clinical parameters and survival of ESCC patients. (A, B, C) The prognostic value of PRRG4, REEP5, and PSMB5 in ESCC patients according to the Kaplan Meier plotter. (D) Binding sites of the miRNA hsa miR 1299 in the target genes, PRRG4, REEP5, and PSMB5. (E, $F, G)$ The relative expression of PRRG4, REEP5, and PSMB5 in primary tumor tissues compared with normal tissues based on the UALCAN database. (H, I, J) The relative expression of PRRG4, REEP5, and PSMB5 was correlated with tumor stages in ESCC. 
There are currently many papers suggesting that the circRNA-miRNA-mRNA axis is related to cancer progression. In this study, we predicted candidate genes targets of miR-1229, among which PRRG4, REEP5, and PSMB5 were associated with a poor prognosis and highly expressed in tumor tissues. The PRRG4 gene can help in the autistic symptoms of WAGR (Wilm's tumor, Aniridia, genitourinary malformations, and mental retardation) syndrome [33]. In addition, Liu et al. identified proline rich and Gla domain 4 (PRRG4) as a potential prognostic marker in cholangiocarcinoma (CCA) [34]. Further study revealed that depletion of receptor expressing enhancing protein 5 (REEP5) significantly reduced growth and invasion in lung cancer [35]. Studies have shown that proteasome subunit beta 5 (PSMB5) is overexpressed in triple-negative breast cancer and significantly associated with a poor prognosis [36] and that knockdown of PSMB5 gene expression inhibits MDA-MB-231 cell growth and migration [37]. These results also suggested that PRRG4, REEP5, and PSMB5 might serve as novel cancer therapeutic targets. Hence, we hypothesized that circ_0000700 may promote the proliferation and migration of ESCC cells by increasing PRRG4, REEP5, and PSMB5 expression. However, in the current study, the specific relationships between miR-1229 and the candidate targets still requires confirmation with a luciferase assay. Therefore, the present study had certain limitations. First, the expression levels and prognostic significance of circ_0000700 and miR-1229 should be evaluated based on clinical samples. Moreover, further functional experiments investigating the relationships among circ_0000700, miR-1229, PRRG4, REEP5, and PSMB5 are suggested in the future to confirm the role of circ_0000700 in this mechanism.

In summary, the present study revealed that hsa_circ_0000700 expression was upregulated in ESCC cells and that silencing hsa_circ_0000700 contributed to restraining the proliferation and migration of ECA-109A and TE-1 cells through binding to miR-1229. Our results suggest that the hsa_circ_0000700/miR-1229/PRRG4-REEP5-PSMB5 axis could be a promising therapeutic target in ESCC.

\section{Abbreviations}

CircRNAs: circular RNAs; ESCC:esophageal squamous cell carcinoma; CCK8: Cell Counting Kit-8; EC: esophageal carcinoma; EAC: esophageal adenocarcinoma; NcRNAs: noncoding RNAs; miRNAs: MicroRNAs; 3'-UTRs: 3'-untranslated regions; ceRNA: competitive endogenous RNA; qRTPCR: quantitative reverse transcription-polymerase chain reaction; NC: negative control; SDS-PAGE: sodium dodecyl sulfate polyacrylamide gel electrophoresis; PVDF: polyvinylidene difluoride; RIP: RNA immunoprecipitation; AGO2: argonaute 2; KEGG: Kyoto Encyclopedia of Genes and Genomes; GO: Gene Ontology; OS: overall survival; SD: standard deviation; WT: wild-type; PRRG4: proline rich and Gla domain 4; CCA: cholangiocarcinoma; REEP5: receptor expressing enhancing protein 5; PSMB5: proteasome subunit beta 5 .

\section{Supplementary Material}

Supplementary figure.

http://www.jcancer.org/v12p2610s1.pdf

\section{Acknowledgements}

This work was supported by grants from the Zhejiang Provincial Natural Science Foundation of China (Grant No's. LSY19A010001, LY19H160004 and LY18H160031) and the Zhejiang Medical and Health Science and Technology Project (Grant No's. 2015122748, 2018260661, 2019326095 and 2019328882).

\section{Availability of data and materials}

The analyzed data sets generated during the study are available from the corresponding author on reasonable request.

\section{Competing Interests}

The authors have declared that no competing interest exists.

\section{References}

1. Shi Y, Guo Z, Fang N, et al. hsa_circ_0006168 sponges miR-100 and regulates mTOR to promote the proliferation, migration and invasion of esophageal squamous cell carcinoma. Biomed Pharmacother. 2019;117:109151.

2. Bray F, Ferlay J, Soerjomataram I, et al. Global Cancer statistics 2018: GLOBOCAN estimates of incidence and mortality worldwide for 36 cancers in 185 countries. CA Cancer J Clin. 2018;68:394-424.

3. Shi N, Shan B, Gu B, et al. Circular RNA circ-PRKCI functions as a competitive endogenous RNA to regulate AKT3 expression by sponging miR-3680-3p in esophageal squamous cell carcinoma. J Cell Biochem. 2019;120:10021-30.

4. $\mathrm{Hu} \mathrm{X}, \mathrm{Wu} \mathrm{D}, \mathrm{He} \mathrm{X}$, et al. circGSK3 $\beta$ promotes metastasis in esophageal squamous cell carcinoma by augmenting $\beta$-catenin signaling. Mol Cancer. 2019;18:160.

5. Wang Q, Zhang Q, Sun H, et al. Circ-TTC17 Promotes Proliferation and Migration of Esophageal Squamous Cell Carcinoma. Dig Dis Sci. 2019;64:751-8.

6. Xing Y, Zha WJ, Li XM, et al. Circular RNA circ-Foxo3 inhibits esophageal squamous cell cancer progression via the miR-23a/PTEN axis. J Cell Biochem. 2020; 121: 2595-2605.

7. Song H, Xu D, Shi P, et al. Upregulated circ RNA hsa_circ_0000337 promotes cell proliferation, migration, and invasion of esophageal squamous cell carcinoma. Cancer Manag Res. 2019;11:1997-2006.

8. Wang Q, Liu H, Liu Z, et al. Circ-SLC7A5, a potential prognostic circulating biomarker for detection of ESCC. Cancer Genet. 2020;240:33-9.

9. Zhang Q, Zhang J, Fu Z, et al. Hypoxia-induced microRNA-10b-3p promotes esophageal squamous cell carcinoma growth and metastasis by targeting TSGA10. Aging (Albany NY). 2019;11:10374-84.

10. Xu Z, Tie X, Li N, et al. Circular RNA hsa_circ_0000654 promotes esophageal squamous cell carcinoma progression by regulating the miR-149-5p/IL-6/ STAT3 pathway. IUBMB Life. 2020; 72: 426-39.

11. Huang $\mathrm{H}$, Wei $\mathrm{L}$, Qin $\mathrm{T}$, et al. Circular RNA ciRS-7 triggers the migration and invasion of esophageal squamous cell carcinoma via miR-7/KLF4 and NF-kB signals. Cancer Biol Ther. 2019;20:73-80.

12. Liu J, Xue N, Guo Y, et al. CircRNA_100367 regulated the radiation sensitivity of esophageal squamous cell carcinomas through miR-217/Wnt3 pathway. Aging (Albany NY). 2019; 11: 12412-27.

13. Shi P, Sun J, He B, et al. Profiles of differentially expressed circRNAs in esophageal and breast cancer. Cancer Manag Res. 2018;10:2207-21. 
14. Dudekula DB, Panda AC, Grammatikakis I, et al. CircInteractome: A web tool for exploring circular RNAs and their interacting proteins and microRNAs. RNA Biology. 2016;13:34-42.

15. Agarwal V, Bell GW, Nam JW, et al. Predicting effective microRNA target sites in mammalian mRNAs. Elife. 2015;4:e05005.

16. Kehl T, Kern F, Backes C, et al. miRPathDB 2.0: a novel release of the miRNA Pathway Dictionary Database. Nucleic Acids Res. 2020;48:D142-7.

17. Chou CH, Shrestha S, Yang CD, et al. miRTarBase update 2018: a resource for experimentally validated microRNA-target interactions. Nucleic Acids Res. 2018;46:D296-D302.

18. Zhou Y, Zhou B, Pache L, et al. Metascape provides a biologist-oriented resource for the analysis of systems-level datasets. Nat Commun. 2019;10:1523.

19. Nagy Á, Lánczky A, Menyhárt O, et al. Validation of miRNA prognostic power in hepatocellular carcinoma using expression data of independent datasets. Sci Rep. 2018;8:9227.

20. Chandrashekar DS, Bashel B, Balasubramanya SAH, et al. UALCAN: A Portal for Facilitating Tumor Subgroup Gene Expression and Survival Analyses. Neoplasia. 2017;19:649-58.

21. Liang $\mathrm{Y}$, Song $\mathrm{X}, \mathrm{Li} \mathrm{Y}$, et al. circKDM4C suppresses tumor progression and attenuates doxorubicin resistance by regulating miR-548p/PBLD axis in breast cancer. Oncogene. 2019;38:6850-66.

22. Wang S, Zhang X, Li Z, et al. Circular RNA profile identifies circOSBPL10 as an oncogenic factor and prognostic marker in gastric cancer. Oncogene. 2019;38:6985-7001.

23. Zheng B, Wu Z, Xue S, et al. hsa_circRNA_100873 upregulation is associated with increased lymphatic metastasis of esophageal squamous cell carcinoma. Oncol Lett. 2019;18:6836-44.

24. Pan Z, Lin J, Wu D, et al. Hsa_circ 0006948 enhances cancer progression and epithelial-mesenchymal transition through the miR-490-3p/HMGA2 axis in esophageal squamous cell carcinoma. Aging (Albany NY). 2019;11:11937-54.

25. He $Y$, Mingyan $E$, Wang $C$, et al. CircVRK1 regulates tumor progression and radioresistance in esophageal squamous cell carcinoma by regulating miR-624-3p/PTEN/PI3K/AKT signaling pathway. Int J Biol Macromol. 2019;125:116-23

26. Dong $\mathrm{Y}, \mathrm{He} \mathrm{D}$, Peng Z, et al. Circular RNAs in cancer: an emerging key player. J Hematol Oncol. 2017;10(1):2.

27. Huang $\mathrm{P}, \mathrm{Qi} \mathrm{B}, \mathrm{Yao} \mathrm{H}$, et al. Circular RNA cSMARCA5 regulates the progression of cervical cancer by acting as a microRNA-432 sponge. $\mathrm{Mol} \mathrm{Med}$ Rep. 2020; 21: 1217-23.

28. Zhou SY, Chen W, Yang SJ, et al. Circular RNA circVAPA regulates breast cancer cell migration and invasion via sponging miR-130a-5p. Epigenomics. 2020; 12: 303-17.

29. Chen Z, Yao N, Gu H, et al. Circular RNA_LARP4 Sponges miR-1323 and Hampers Progression of Esophageal Squamous Cell Carcinoma Through Modulating PTEN/PI3K/AKT Pathway. Dig Dis Sci. 2020;65:2272-83.

30. Zhang L, Bu Z, Shen J, et al. A novel circular RNA (hsa_circ_0000370) increases cell viability and inhibits apoptosis of FLT3-ITD-positive acute myeloid leukemia cells by regulating miR-1229 and S100A7A. Biomed Pharmacother. 2020;122:109619.

31. Lin L, Cai J. Circular RNA circ-EGLN3 promotes renal cell carcinoma proliferation and aggressiveness via miR-1229-mediated IRF7 activation. J Cell Biochem. 2020;10.1002/jcb.29620.

32. Meng L, Liu S, Ding P, et al. Circular RNA ciRS-7 inhibits autophagy of ESCC cells by functioning as miR-1229 sponge to target EGFR signaling. J Cell Biochem. 2020;121:1039-49.

33. Justice ED, Barnum SJ, Kidd T. The WAGR syndrome gene PRRG4 is a functional homologue of the commissureless axon guidance gene. PLoS Genet. 2017;13:e1006865.

34. Liu J, Liu W, Li H, et al. Identification of key genes and pathways associated with cholangiocarcinoma development based on weighted gene correlation network analysis. PeerJ. 2019;7:e7968.

35. Park CR, You DJ, Park S, et al. The accessory proteins REEP5 and REEP6 refine CXCR1-mediated cellular responses and lung cancer progression. Sci Rep. 2016;6:39041.

36. Wei W, Zou Y, Jiang Q, et al. PSMB5 is associated with proliferation and drug resistance in triple-negative breast cancer. Int J Biol Markers. 2018;33:102-8.

37. Wang CY, Li CY, Hsu HP, et al. PSMB5 plays a dual role in cancer development and immunosuppression. Am J Cancer Res. 2017;7:2103-20. 